\title{
Community Engagement among the BioSense 2.0 User Group
}

\author{
Stacey Hoferka*1, Marcus Rennick², Erin E. Austin ${ }^{3}$, Anne Burke ${ }^{4}$, Rosa Ergas ${ }^{5}$, Jay \\ Fiedler $^{6}$, Laura Streichert ${ }^{7}$ and Amanda Wahnich ${ }^{3}$
}

${ }^{1}$ IL Dept Public Health, Chicago, IL, USA; ${ }^{2}$ ASTHO, Arlington, VA, USA; ${ }^{3}$ Virginia Dept. of Health, Richmond, VA, USA; ${ }^{4}$ Utah Dept. of

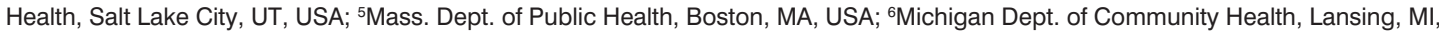
USA; ${ }^{7}$ ISDS, Brighton, MA, USA

\section{Objective}

This roundtable will provide a forum for the syndromic surveillance Community of Practice (CoP) to learn about activities of the BioSense 2.0 User Group (BUG) workgroups that address priority issues in syndromic surveillance. It will be an opportunity to discuss key challenges faced by public health jurisdictions in the era of Meaningful Use and identify further needs and best practices in the areas of data quality, data sharing, onboarding, and developing syndrome definitions.

\section{Introduction}

BioSense 2.0 has become a platform for technical receipt and analysis of syndromic surveillance data for many jurisdictions nationwide, as well as a collaborative effort that has engaged a larger community of syndromic surveillance practitioners, Governance Group, and federal agencies and organizations. The potential longterm benefits of BioSense 2.0 for resource and data sharing have at times been overshadowed by the short-term limitations of the system and disconnected efforts among the CoP. In May 2014, representatives from 41 jurisdictions attended a 2-day, in-person meeting where four workgroups were formed to address on-boarding, data quality, data sharing and syndrome definition in an effort to advance changes that resonate with actual surveillance practice.

\section{Description}

Representatives from each workgroup will describe their efforts to date and engage the $\mathrm{CoP}$ in the discussion. The goals of the workgroups are to coordinate efforts nationwide, better inform development of BioSense 2.0 to the Governance Group and CDC, and achieve high-quality outcomes for the practice of syndromic surveillance. As Figure 1 illustrates, the BioSense 2.0 workgroups create a cooperative environment to address complex system-wide concerns. The workgroups' focuses are described as follows:

Data quality workgroup aims to document data processing steps, develop tools to assess data in BioSense 2.0, and determine best practices for addressing data quality deficiencies. Working toward these aims will give jurisdictions the ability to understand, measure, and improve their data, resulting in increased comparability, utility and trust of data housed in the BioSense 2.0 application and output derived from these data.

Data sharing workgroup is rooted in the fundamental desire to leverage cloud based data storage of the BioSense 2.0 architecture toward enhanced data sharing within and between jurisdictions and system participants. The two primary initiatives of this group are to specify data sharing roles and permissions for users in the system and to evaluate the need for Data Use Agreements between jurisdictions to facilitate data sharing activities.

On-boarding workgroup aims to create processes for and implement documentation of on-boarding standards and contextualize these data through creation of jurisdictional profiles. These efforts will help to define functionality for automated structural message conformance and content, ensure compliance to implementation guides, and describe best practices through a reference mapping process.

Syndrome definition workgroup aims to standardize the syndrome definitions available in BioSense 2.0 and determine the best processes for syndrome binning. Working towards these aims will ensure that data are accurately parsed and searchable, resulting in comparability across jurisdictions.

\section{Audience Engagement}

Activities from each of the four workgroups will be presented to the audience. Current status, planned development, and functionality requests to the $\mathrm{CDC}$ will be identified.Participants will be asked to comment on benefits and additional needs that would improve the current work. Each workgroup will spend five minutes presenting a tool or solution and then invite open discussion with the audience. The end result of the roundtable will be a list of functionality and enhancement requirements to be considered in the workgroups' ongoing efforts. Recruitment of additional workgroup participants will also occur.

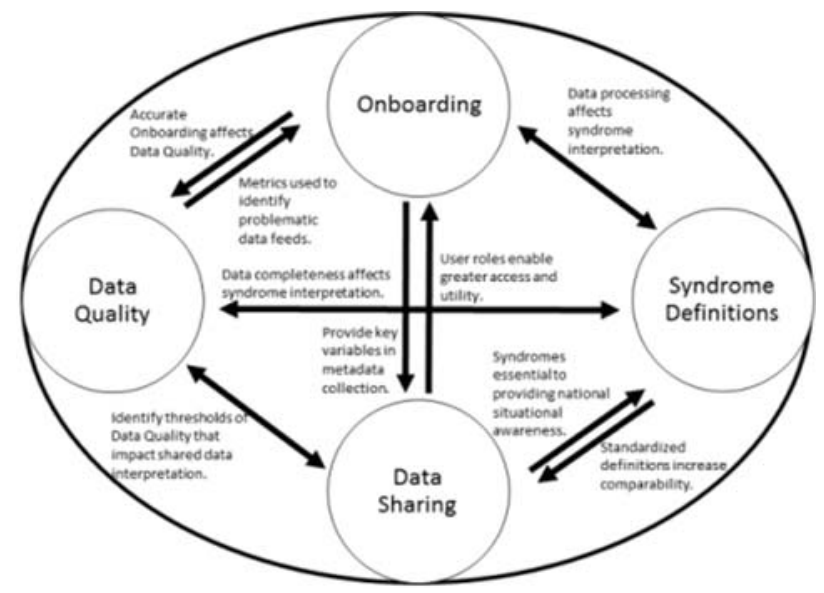

Figure 1. BioSense 2.0 User Group Community of Practice Workgroups

\section{Keywords}

BioSense 2.0; Workgroups; Community of Practice; Syndromic Surveillance

*Stacey Hoferka
E-mail: stacey.hoferka@illinois.gov

